\title{
BUZANO'S INEQUALITY AND BOUNDS FOR ROOTS OF ALGEBRAIC EQUATIONS
}

\author{
MASATOSHI FUJII AND FUMIO KUBO
}

(Communicated by Paul S. Muhly)

Dedicated to Professor Tsuyoshi Ando on his 60th birthday

ABstract. A new bound for roots of algebraic equations will be given as a consequence of an inequality due to Buzano.

\section{INTRODUCTION}

Buzano [1] obtained an extension of Schwarz's inequality: If $\mathbf{a}, \mathbf{b}, \mathbf{x}$ are vectors in an inner product space $\mathscr{H}$, then

$$
|\langle\mathbf{a} \mid \mathbf{x}\rangle \cdot\langle\mathbf{x} \mid \mathbf{b}\rangle| \leq \frac{\|\mathbf{a}\| \cdot\|\mathbf{b}\|+|\langle\mathbf{a} \mid \mathbf{b}\rangle|}{2}\|\mathbf{x}\|^{2} .
$$

Since her proof is a little complicated, a new, simple proof will be given with the equality condition.

Let $P$ be an orthogonal projection on a subspace of an inner product space $\mathscr{H}$. If $\mathbf{a}, \mathbf{b} \in \mathscr{H}$, then the usual Schwarz's inequality implies that

$$
|\langle(2 P-I) \mathbf{a} \mid \mathbf{b}\rangle| \leq\|\mathbf{a}\| \cdot\|\mathbf{b}\| .
$$

Let $(\mathbf{u} \otimes \mathbf{v}) \mathbf{w}=\langle\mathbf{w} \mid \mathbf{v}\rangle \mathbf{u} \quad(\mathbf{w} \in \mathscr{H})$. Then the operator $\mathbf{x} \otimes \mathbf{x}$ is an orthogonal projection if $\|\mathbf{x}\|=1$, and hence $|\langle(2 \mathbf{x} \otimes \mathbf{x}-I) \mathbf{a} \mid \mathbf{b}\rangle| \leq\|\mathbf{a}\| \cdot\|\mathbf{b}\|$, which implies the required one:

$$
2|\langle(\mathbf{x} \otimes \mathbf{x}) \mathbf{a} \mid \mathbf{b}\rangle|-|\langle\mathbf{a} \mid \mathbf{b}\rangle| \leq|\langle(2 \mathbf{x} \otimes \mathbf{x}-I) \mathbf{a} \mid \mathbf{b}\rangle| \leq\|\mathbf{a}\| \cdot\|\mathbf{b}\| .
$$

The equality holds iff two inequality signs in the last line turn out to be equal, from which one obtains the equality condition: The equality in (1) holds if

$$
\mathbf{x}= \begin{cases}\alpha\left(\frac{\mathbf{a}}{\|\mathbf{a}\|}+\frac{\langle\mathbf{a} \mid \mathbf{b}\rangle}{|\langle\mathbf{a} \mid \mathbf{b}\rangle|} \frac{\mathbf{b}}{\|\mathbf{b}\|}\right), & \text { when }\langle\mathbf{a} \mid \mathbf{b}\rangle \neq 0, \\ \alpha\left(\frac{\mathbf{a}}{\|\mathbf{a}\|}+\beta \frac{\mathbf{b}}{\|\mathbf{b}\|}\right), & \text { when }\langle\mathbf{a} \mid \mathbf{b}\rangle=0,\end{cases}
$$

Received by the editors June 15, 1990.

1980 Mathematics Subject Classification (1985 Revision). Primary 47A12; Secondary 26C10.

Key words and phrases. Numerical radius companion matrix, Schwarz's inequality bound for roots.

This research was partially supported by Grant-in-Aid for Scientific Research, Ministry of Education. 
where $\alpha, \beta$ are complex numbers with $|\beta|=1$.

Define the numerical radius $w(T)$ of an operator $T$ acting on $\mathscr{H}$ by

$$
w(T)=\sup \{|\langle T \mathbf{x} \mid \mathbf{x}\rangle|:\|\mathbf{x}\|=1\} .
$$

Thus Buzano's inequality with the equality condition implies at once the following theorem.

Theorem 1. If $T=\mathbf{a} \otimes \mathbf{b}$ is a linear operator of rank one, then

$$
w(T)=\frac{\|\mathbf{a}\| \cdot\|\mathbf{b}\|+|\langle\mathbf{a} \mid \mathbf{b}\rangle|}{2} .
$$

In this paper, Theorem 1 will be applied to obtain a bound for roots of algebraic equations. Other comments on Buzano's inequality will be published elsewhere.

\section{BOUNDS FOR ROOTS OF ALGEBRAIC EQUATIONS}

Let

$$
C=\left(\begin{array}{ccccc}
-a_{n-1} & -a_{n-2} & \cdots & -a_{1} & -a_{0} \\
1 & 0 & \cdots & 0 & 0 \\
0 & 1 & \ddots & 0 & 0 \\
\vdots & \vdots & \ddots & & \vdots \\
0 & 0 & & 1 & 0
\end{array}\right)
$$

be the companion matrix associated with the algebraic equation

$$
z^{n}+a_{n-1} z^{n-1}+a_{n-2} z^{n-2}+\cdots+a_{1} z+a_{0}=0 .
$$

It is well known (cf. [5]) that the set of roots of (3) is identical with the spectrum $\sigma(C)$ of $C$. In [3], it was shown that those classical bounds for roots were obtained as operator norms of the companion matrix $C$ (cf. [4]). Since the numerical range $W(T)=\{\langle T \mathbf{x} \mid \mathbf{x}\rangle:\|\mathbf{x}\|=1\}$ contains $\sigma(T)$, it is expected that an estimation of $w(C)$ gives a new bound for roots of (3).

Theorem 2. If $z$ is a root of an algebraic equation (3) then

$$
|z| \leq \cos \frac{\pi}{n+1}+\frac{\sqrt{\sum_{i=0}^{n-1}\left|a_{i}\right|^{2}}+\left|a_{n-1}\right|}{2} .
$$

Proof. Since $C=S-\mathbf{e}_{1} \otimes \mathbf{a}$, where

$$
\mathbf{a}=\left(\begin{array}{c}
\overline{a_{n-1}} \\
\overline{a_{n-2}} \\
\vdots \\
\overline{a_{0}}
\end{array}\right), \quad \mathbf{e}_{1}=\left(\begin{array}{c}
1 \\
0 \\
\vdots \\
0
\end{array}\right), \quad \text { and } \quad S=\left(\begin{array}{cccccc}
0 & 0 & 0 & \cdots & 0 & 0 \\
1 & 0 & 0 & \cdots & 0 & 0 \\
0 & 1 & 0 & \cdots & 0 & 0 \\
& \cdots & & \cdots & & \cdots \\
0 & 0 & 0 & \cdots & 1 & 0
\end{array}\right) \text {, }
$$

one has only to estimate the value

$$
w(C)=w\left(S-\mathbf{e}_{1} \otimes \overline{\mathbf{a}}\right) \leq w(S)+w\left(\mathbf{e}_{1} \otimes \overline{\mathbf{a}}\right)=w(S)+\frac{\|\mathbf{a}\|+\left|a_{n-1}\right|}{2} .
$$

To estimate $w(S)$, one can consult with the recent paper of Davidson and Holbrook [2].

Finally a comparison with the bound due to Carmichael-Mason (cf. [5]) will be given: If $z$ is a root of (3) then $|z| \leq B_{C M}=\sqrt{1+\sum_{i=0}^{n-1}\left|a_{i}\right|^{2}}$. Their bound 
is not always better than the one in Theorem 2, and vice versa. It is obvious that if the second leading coefficient vanishes and $\|\mathbf{a}\|$ is fairly large, then the new bound is better than $B_{C M}$.

\section{REFERENCES}

1. M. L. Buzano, Generalizzatione della diseguaglianza di Cauchy-Schwarz, Rend. Sem. Mat. Univ. e Politech. Trimo 31 (1971/73), 405-409.

2. K. R. Davidson and J. A. R. Holbrook, Numerical radii of zero-one matrices, Michigan Math. J. 35 (1988), 261-267.

3. M. Fujii and F. Kubo, Operator norms as bounds for roots of algebraic equations, Proc. Japan Acad. Sci. 49 (1973), 805-808.

4. R. A. Horn and C. A. Johnson, Matrix analysis, Cambridge Univ. Press, Cambridge, 1985.

5. M. Marden, The geometry of zeros of a polynomial in a complex variable, 2 nd ed., Math. Survey, vol. 3, Amer. Math. Soc., Providence, RI, 1960.

Department of Mathematics, Osaka Kyoiku University, Tennoji, Osaka 543, Japan

Department of Mathematics, Faculty of Science, Toyama University, Gofuku, TOYAMA 930, JAPAN

E-mail address: E01315@SINET.AD.JP 